\title{
Potential Pathogenicity of Candida Species Isolated from Oral Cavity of Patients with Diabetes Mellitus
}

\author{
Hasti Nouraei $\left(\mathbb{D},{ }^{1}\right.$ Mehdi Ghaderian Jahromi, ${ }^{2}$ Leila Razeghian Jahromi, ${ }^{3}$ \\ Kamiar Zomorodian, ${ }^{1,4}$ and Keyvan Pakshir ${ }^{1,4}$ \\ ${ }^{1}$ Department of Parasitology and Mycology, School of Medicine, Shiraz University of Medical Sciences, Shiraz, Iran \\ ${ }^{2}$ Department of Radiology, School of Medicine, Medical Imaging Research Center, Shiraz University of Medicine Sciences, Shiraz, Iran \\ ${ }^{3}$ Department of Psychiatry, School of Medicine, Shiraz University of Medicine Sciences, Shiraz, Iran \\ ${ }^{4}$ Basic Sciences in Infectious Diseases Research Center, School of Medicine, Shiraz University of Medical Sciences, Shiraz, Iran
}

Correspondence should be addressed to Keyvan Pakshir; pakshirk@gmail.com

Received 3 March 2021; Revised 18 April 2021; Accepted 12 May 2021; Published 27 May 2021

Academic Editor: Himanshu Garg

Copyright (C) 2021 Hasti Nouraei et al. This is an open access article distributed under the Creative Commons Attribution License, which permits unrestricted use, distribution, and reproduction in any medium, provided the original work is properly cited.

Introduction. In the recent decade, the increased immunocompromised population such as diabetic patients makes a high incidence of invasive Candida infections. Diabetes mellitus is the most common endocrine metabolic disorder, and diabetic patients are more susceptible to oral candidiasis infection. Candidiasis is an opportunistic fungal infection caused by many species of Candida. Secretion of exoenzymes plays an important role in the virulence and pathogenesis of Candida species. The aim of this study was to evaluate the potential role of phospholipase, esterase, and hemolytic activity of Candida species isolated from oral cavity lesions of diabetic patients. Methods. A total of 108 Candida species including 75 Candida albicans and 33 non-Candida albicans species were recovered from the oral cavity of diabetic patients included in our study. Egg yolk agar, Tween 80 opacity medium, and blood agar plate assays were used for determining phospholipase, esterase, and hemolytic activities, respectively. Results. Candida albicans species had the most exoenzyme activity in comparison to non-albicans isolates. Candida albicans isolates showed $97.3 \%, 100 \%$, and 77.3\% phospholipase, hemolysin, and esterase activities, respectively. The difference between Candida albicans and non-Candida albicans was significant in phospholipase $(P<0.001)$ and hemolytic activity $(P=0.027)$, but not significant in esterase activity $(P=0.076)$. Conclusion. This study showed that most of the isolates had different enzymatic patterns, and Candida albicans isolates had the most exoenzyme activity. So due to the potential effects of these enzymes in pathogenesis and virulence effects of Candida species, we can conclude that the severity of extracellular enzymes may play a role in the severity of signs and symptoms of Candida oral cavity infections in diabetic patients.

\section{Introduction}

Diabetes mellitus (DM) is one of the most prevalent metabolic chronic disorders throughout the world caused by the dysfunction of $\beta$ cells in pancreatic islet 1 in which glucose plasma levels remain high for a long period $[1,2]$. Among many reasons that caused the high morbidity of these diseases, chronic macro- and microvascular complications such as neuropathy, nephropathy, retinopathy, and heart complications are more important [3]. The rates of this disease are equal in both genders and affect more than 425 million people all around the world [4]. In the 21st century, DM is becoming a global epidemic and one of the largest emerging threats to public health which significantly affect the quality of life and longevity of the patients, as well as healthcare costs $[5,6]$.

The overall prevalence of diabetes among adults over 18 years old has increased during the past decades, and the World Health Organization (WHO) predicts that this will increase to 439 million, almost $10 \%$ of adults in 2030 [7]. Diabetes mellitus is usually associated with systemic complications, such as kidney disease, eye disease, recurrent fungal skin infection, and oral diseases, including gingivitis, periodontitis, and candidiasis $[2,8]$.

Diabetes mellitus is divided into two types: insulindependent (type 1) and non-insulin-dependent (type 2). Ninety percent of people have type 2 diabetes mellitus 
(DM2) that mainly has correlation with personal lifestyle, including high-calorie diets, low physical activity, and smoking [9].

Diabetes mellitus has many oral manifestations, complication, and infections such as dry mouth, burning mouth syndrome (BMS), taste disorders, oral candidiasis, rhinocerebral zygomycosis (mucormycosis), aspergillosis, geographic tongue, oral lichen planus, delayed wound healing, periodontal disease, gingivitis, and increased incidence of infection [10].

Yeast infections in diabetic patients are common [11]. Candida spp. is frequently found in patients with poor glycemic control, and it was already shown that increased oral Candida carriage is related to increasing levels of glucose in saliva [12]. Oral candidiasis is commonly caused by C. albicans species and presents many clinical forms (pseudomembranous candidiasis, cheilitis, and stomatitis) [13].

Some factors have a major influence on the balance between host and yeasts and caused the transition of Candida sp. (C. albicans, C. glabrata, C. tropicalis, C. parapsilosis, and C. krusei) [14] from commensal to pathogen and causing infection such as reduced salivary flow, higher salivary glucose levels, and impaired candidacidal activity of neutrophils [15]. The risk factors for oral candidiasis are complex, but it is known that tongue lesions, tobacco smoking, alcohol consumption, denture wearing, the intake of medication, and immunosuppression (e.g., diabetes mellitus) clearly influence oral candidiasis [16]. However, in patients with DM that are immunocompromised, these species can cause invasive infections. Several studies have shown the link between Candida sp. infection and DM [17]. Higher expressions in enzymatic activity and ability of biofilm formation in Candida spp. are two of the most important features in oral candidiasis [18].

Many studies have established a relationship between hydrolytic enzyme activity and an increase in the pathogenic ability of Candida spp. [6, 19].

Due to higher blood glucose concentration in diabetic patients, Candida spp. isolates present significantly higher hemolytic, esterase, and phospholipase enzymatic activity $[20,21]$. Another hypothesis was that these species are more pathogenic in DM because of abnormal conditions [22]. They also can cause inflammatory reactions by increasing vascular permeability and clinical symptoms, which may disturb the humoral host defense [23]. These enzymes could digest and damage the membrane, initiating cell lysis and facilitating the penetration of the infecting fungi [24].

So, due to this fact that the presence of high enzymatic activity in Candida species oral candidiasis could exacerbate different clinical symptoms in DM patients, we decided to design this study to evaluate this potential pathogenicity of Candida species isolated from the oral cavity of patients with diabetes mellitus.

\section{Materials and Methods}

2.1. Yeast Isolates. In this study, a total of 108 stock Candida species consisting of 75 Candida albicans and 33 non-albicans Candida (NAC) species including 16 C. dubliniensis, 9
C. glabrata, 3 C. parapsilosis, 2 C. guilliermondii, 1 C. tropicalis, 1 C. krusei, and 1 C. kefyr which previously were recovered from diabetic patients were used. These species were identified to the species level based on results of conventional methods such as the ability to produce germ tubes, chlamydoconidia production in corn meal agar medium, and colony color on chromogenic media (CHROMagar Candida) and molecular method (PCR-RFLP) [25].

2.2. Determination of Phospholipase Activity. Candida species isolates were screened for phospholipase activity by measuring the size of the zone of precipitation after growth on egg yolk agar [26]. The egg yolk medium consisted of $58.4 \mathrm{~g} \mathrm{NaCl}$ and $5.5 \mathrm{~g} \mathrm{CaCl}_{2}$ and $65 \mathrm{~g}$ Sabouraud dextrose agar and $2 \%$ sterile egg yolk.

First, the components without the egg yolk were mixed and sterilized; then, the egg yolk was centrifuged at 5,000 $\times$ $\mathrm{g}$ for $30 \mathrm{~min}$, and $20 \mathrm{ml}$ of the supernatant was added to the sterilized medium. The inocula were prepared (McFarland 2 turbidity) and were deposited onto the egg yolk agar medium and left to dry at room temperature. Each culture was then incubated at $37^{\circ} \mathrm{C}$ for 10 days, after which the diameter of the precipitation zone $(\mathrm{Pz})$ was determined. Phospholipase activity was expressed as the ratio of the diameter of the colony to the diameter of the colony plus the precipitation zone (in $\mathrm{mm}$ ) [26].

2.3. Determination of Esterase Activity. Esterase activity was measured using the Tween 80 opacity test medium [27], which was prepared with $10 \mathrm{~g}$ of bacteriological peptone (Merck, Germany), $5 \mathrm{~g}$ of sodium chloride, $0.1 \mathrm{~g}$ of calcium chloride, $15 \mathrm{~g}$ of agar, and $1000 \mathrm{ml}$ of distilled water. After, the medium was autoclaved; then, $5 \mathrm{ml}$ of autoclaved Tween 80 was added (Merck, Germany). Then, 10 microlitres of Candida suspension $\left(10^{6}\right.$ cells $\left./ \mathrm{ml}\right)$ was inoculated as a spot and was incubated at $30^{\circ} \mathrm{C}$ for 10 days. The colony diameter (a) and the diameter of colony plus precipitation zone $(b)$ were measured. The esterase activities were expressed as Ez value $(a / b)$ as described by Price et al. [26].

2.4. Determination of Hemolysin Activity. Hemolytic activity was evaluated with a blood plate assay [28]. Sheep blood SDA were prepared by adding $7 \mathrm{ml}$ of fresh sheep blood to $100 \mathrm{ml}$ of SDA supplemented with 3\% glucose. Suspensions equal to McFarland 2 turbidity from the pure culture of the yeast colonies were prepared. Ten microliters of this suspension was spotted on prepared media and incubated at $37^{\circ} \mathrm{C}$ for $48 \mathrm{~h}$. For hemolytic activity, a ring of lysis around the colonies was considered complete (in case of a totally translucent ring; beta), incomplete (in case of greenish-black halo; alpha), and no hemolysis (gamma or none). Candida albicans ATCC10261 was used as a control for all three methods.

2.5. Statistical Analysis. Results were analyzed using the SPSS22 (Statistical Package for the Social Sciences) program. The Fisher exact test was considered for statistical significance with a $P$ value less than 0.05 .

2.6. Ethical Approval. This project was found to be in accordance with the ethical principles and the national norms and 
TABLE 1: Comparisons and distribution of virulence factors of clinical Candida species isolated from diabetic patients.

\begin{tabular}{lcccccccccc}
\hline \multirow{2}{*}{ Species $(n)$} & \multicolumn{4}{c}{ Phospholipase } & \multicolumn{4}{c}{ Esterase } \\
& Neg & $1+$ & $2+$ & $3+$ & $4+$ & Neg & $1+$ & $2+$ & $3+$ & $4+$ \\
\hline C. albicans (75) & $2(2.7 \%)$ & $0(0 \%)$ & $1(1.3 \%)$ & $3(4 \%)$ & $69(92 \%)$ & $17(22.6 \%)$ & $0(0 \%)$ & $1(1.3 \%)$ & $5(6.7 \%)$ & $52(69.4 \%)$ \\
Non-Candida albicans (33) & $11(33.4 \%)$ & $0(0 \%)$ & $0(0 \%)$ & $2(6 \%)$ & $20(60.6 \%)$ & $12(36.5 \%)$ & $0(0 \%)$ & $1(3 \%)$ & $3(9 \%)$ & $17(51.5 \%)$ \\
\hline
\end{tabular}

standards for conducting medical research in Iran and has been approved by the research ethics committee (IR. SUMS.REC.1390.3755).

\section{Results}

3.1. Phospholipase Activities of Candida spp. In total, out of 75 samples of Candida albicans, 73 (97.3\%) isolates had phospholipase activity, and 69 species (92\%) showed +4 positive for phospholipase activity and only 2 isolates $(2.7 \%)$ had no phospholipase activity. However, out of 33 non-Candida albicans samples, only $20(60.6 \%)$ isolates had +4 positive phospholipase activity and 11 (33.3\%) isolates had no phospholipase activity. Among non-Candida albicans samples, all of the $C$. dubliniensis isolates showed +4 positive phospholipase activities (100\%), but C. krusei and C. guilliermondii had no phospholipase activity. More details are explained in Table 1. There were statistically significant differences in phospholipase activity between two groups of $C$. albicans and non-albicans $(P<0.001)$.

3.2. Esterase Activities of Candida spp. Out of 75 samples of $C$. albicans, 58 (77.3\%) isolates had esterase activity, and 52 isolates $(69.3 \%)$ showed +4 positive esterase activity. On the other hand, 17 isolates (22.7\%) had no esterase activity. Among non-Candida albicans species, 21 (63.6\%) isolates had esterase activity and 17 (51.51\%) isolates showed +4 positive esterase activities (Table 1). There was no significant statistical correlation between the two groups of Candida albicans and non-albicans species in esterase activities $(P=0.07)$.

3.3. Hemolytic Activities of Candida spp. All of the 75 studied samples of C. albicans (100\%) had beta-hemolytic activity. Out of the 33 samples of non-Candida albicans, 30 isolates (91\%) had beta-hemolytic activity and 3 isolates (9\%) had alpha-hemolytic activity that included 1 C. dubliniensis and 2 C. glabrata. None of the isolates had gamma-hemolytic activity. Statistical analysis for production of hemolysin between $C$. albicans species and non-Candida albicans species showed that there was a significant difference between 2 species $(P=0.027)$ (Figure 1$)$.

\section{Discussion}

Diabetes mellitus characterized by hyperglycemia resulting from reducing insulin secretion, insulin action, or both is an endocrine disease that is a global health problem [29]. An increasing level of glucose in serum causes variation damage to different types of cells, such as endothelial cells, neurons, renal cells, and keratinocytes [30]. An increasing glucose level in serum could damage monocyte and neutro- phil adherence, chemotaxis, and phagocytosis which are important in host defense. During Candida infection, raising glucose in the cell causes increasing adherence and invasion of organisms to the infected tissues [31]. Oral candidiasis can be diagnosed by the differential patterns of mucosal changes like erythematous, pseudomembranous, and curdlike plaques [32].

A previous study showed increases in the prevalence of $C$. albicans strains in the oral cavity of patients with DM. In another study, this prevalence rate of the Candida species was $87.5 \%$ [33]. Fatahinia et al. demonstrated that the prevalence of $C$. albicans isolates in the oral cavity of diabetic patients was significantly greater than that of nondiabetic patients $(66.7 \%$ and $57.1 \%)$, and also, they reported that there were some differences in the pattern of phospholipase, esterase, and hemolysin secretion between these 2 groups. For example, hemolytic activity was detected in $100 \%$ of isolates in the diabetic group, but this rate was $52 \%$ of isolates in the nondiabetic patient groups [21].

Many factors in Candida spp have been attributed to increasing the incidence of candidiasis in diabetic patients such as enzymatic activity, biofilm formation, hydrophobicity, phenotypic switching, and yeast-to-hypha transition [24]. Producing extracellular enzymes by Candida spp can play an important role in its pathogenicity, invasion, and destroying host tissues and effect on developing clinical signs. Candida spp also used their phospholipase and esterase enzymes for the invasion to host tissue and hemolysin for lysing the blood cell. Although Candida has the ability to produce these enzymes, the amount and strength of these enzymes are various among different species, and because of the different sources of their isolation, they have different patterns of secretion [34].

Phospholipase secretions also help the organism to penetrate better to host tissue [24]. In the present study, phospholipase activity was detected in $73(97.3 \%)$ out of the $75 \mathrm{C}$. albicans isolates and in $22(66.6 \%)$ of NCA. These results demonstrate that the group of $C$. albicans isolates produce high phospholipase enzyme which is very important for damaging tissue. Tsang et al. [20] reported that $100 \%$ of $C$. albicans-tested isolates showed phospholipase activity that their result was close to our result. This enzyme increases the ability of organisms for the destruction of immune factors of host cells and makes better opportunity for invasion and achieving nutrients [35]. Esterase activity is another virulence factor in Candida spp which digests the surface membrane of the host cell and causes better binding, penetration, and invasion, thus playing important roles in Candida pathogenicity [21].

In this study, $77.3 \%$ of C. albicans isolates and $63.5 \%$ of NCA showed esterase activity which was almost close 


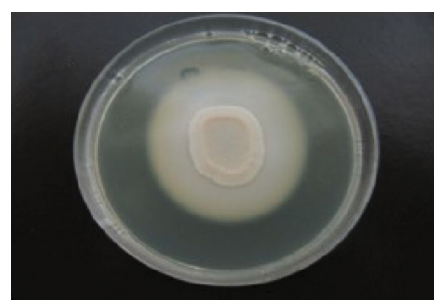

(a)

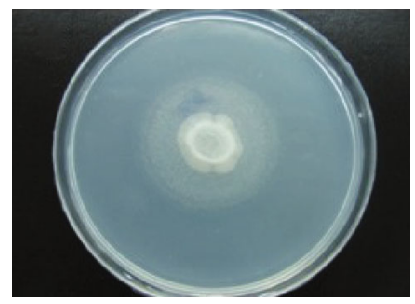

(b)

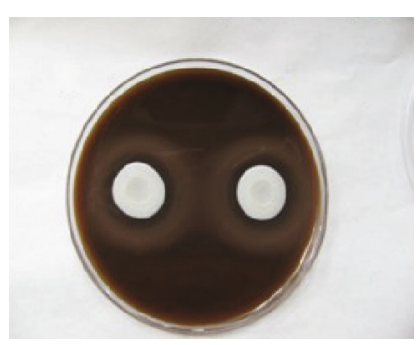

(c)

FIgure 1: (a) Phospholipase production of Candida spp. isolates on egg yolk agar. (b) Positive activity of esterase in Candida species. (c) Showed beta-hemolytic activity.

between the two groups while Fatahinia et al. [21] reported that only $21.6 \%$ of their C. albicans isolates presented this activity, and this data was not in agreement with our result. The amount of esterase secretion could be considered an important factor in tissue invasion for organisms, and it could exacerbate symptoms in diabetic patients [36].

The other virulence factor that is important in the pathogenicity of Candida spp is the hemolysin enzyme that facilitates the pathogen to extract iron from molecules with hemoglobin or hemin [24]. In our study, $100 \%$ C. albicans of isolates had hemolytic activity; our data was in agreement with Fatahinia et al. [21] results but not in agreement with Tsang et al. [20] study by which the hemolytic activity was $100 \%$ and $80 \%$ of isolates, respectively. The presence of white and secreted plaque lesions in oral candidiasis (thrush) and also serous fluid in lesions and cheilitis (perleche) may link with the number of blood cells in the mouth and covered oral mucosa so this condition can make facility in Candida species hemolysin secretion and obtain iron from the blood.

Luo et al. believed that increased hemolysin activity among Candida spp isolates in DM patients was because of increasing blood glucose concentration, and also, an increased salivary glucose concentration in DM patients may contribute directly or indirectly to rising hemolysin production in Candida spp. [37]. This is another reason for the importance of this enzyme.

According to this fact that one of the important pathogenic factors in Candida species is their extracellular enzymes and due to the weakness of the defense barrier in diabetic patients, it can be guessed that the production of these enzymes plays an important role in severity of invasion to the mucosal cell. So, among Candida species isolated from oral lesions, if the production of these enzymes is more intense, the severity and extent of the lesion will probably be higher.

Here, due to the high production of enzymes in Candida albicans isolates, it can be inferred that the incidence of oral mucosal complications with these species is probably higher than other species and more enzyme production by these species may be one of the factors that cause these species to be more isolated from oral mucosal lesions.

\section{Conclusion}

Diabetes is a chronic health condition, and diabetic patients are more susceptible to oral candidiasis. Virulence factors like phospholipase, esterase, and hemolysin activity play an important role in the pathogenicity of Candida species. Knowledge about these virulence factors can be helpful in better management of these largely opportunistic fungal infections and treatment of disease. Further research aimed at characterizing the other risk factors of Candida species is needed for better management, preventive therapy, and treatment of diabetes mellitus.

\section{Data Availability}

The data used to support the findings of this study were supplied by Shiraz University of Medical Sciences under license and so cannot be made freely available. Requests for access to these data should be made to Keyvan Pakshir, pakshirk@ gmail.com.

\section{Disclosure}

This study was extracted from the MD thesis by Mehdi Ghadarian Jahromi.

\section{Conflicts of Interest}

The authors report no conflicts of interest.

\section{Acknowledgments}

This study was funded by the Deputy of Research and Technology of Shiraz University of Medical Sciences, Shiraz, Iran (Grant No. 3755).

\section{References}

[1] M. Bakhti, A. Böttcher, and H. Lickert, "Modelling the endocrine pancreas in health and disease," Nature Reviews Endocrinology, vol. 15, no. 3, pp. 155-171, 2019.

[2] L. Martorano-Fernandes, L. M. Dornelas-Figueira, R. M. Marcello-Machado et al., "Oral candidiasis and denture stomatitis in diabetic patients: systematic review and meta-analysis," Brazilian Oral Research, vol. 34, 2020.

[3] E. W. Gregg, N. Sattar, and M. K. Ali, "The changing face of diabetes complications," The lancet Diabetes \& endocrinology, vol. 4, no. 6, pp. 537-547, 2016.

[4] C. D. Mathers and D. Loncar, "Projections of global mortality and burden of disease from 2002 to 2030," PLoS Medicine, vol. 3, no. 11, article e442, 2006. 
[5] A. Karaa and A. Goldstein, "The spectrum of clinical presentation, diagnosis, and management of mitochondrial forms of diabetes," Pediatric Diabetes, vol. 16, no. 1, pp. 1-9, 2015.

[6] C. F. Rodrigues, M. E. Rodrigues, and M. Henriques, "Candida sp. infections in patients with diabetes mellitus," Journal of Clinical Medicine, vol. 8, no. 1, p. 76, 2019.

[7] E. Mauri-Obradors, A. Estrugo-Devesa, E. Jane-Salas, M. Vinas, and J. Lopez-Lopez, "Oral manifestations of diabetes mellitus. A systematic review," Medicina Oral, Patología Oral y Cirugía Bucal, vol. 22, no. 5, article e586, 2017.

[8] M. R. Knop, T. T. Geng, A. W. Gorny et al., "Birth weight and risk of type 2 diabetes mellitus, cardiovascular disease, and hypertension in adults: a meta-analysis of 7646267 participants from 135 studies," Journal of the American Heart Association, vol. 7, no. 23, article e008870, 2018.

[9] Z. Tao, A. Shi, and J. Zhao, "Epidemiological perspectives of diabetes," Cell Biochemistry and Biophysics, vol. 73, no. 1, pp. 181-185, 2015.

[10] D. A. Albert, A. Ward, P. Allweiss et al., "Diabetes and oral disease: implications for health professionals," Annals of the New York Academy of Sciences, vol. 1255, no. 1, pp. 1-15, 2012.

[11] J. Casqueiro, J. Casqueiro, and C. Alves, "Infections in patients with diabetes mellitus: a review of pathogenesis," Indian journal of endocrinology and metabolism, vol. 16, no. 7, p. 27, 2012.

[12] S. Kumar, S. Padmashree, and R. Jayalekshmi, "Correlation of salivary glucose, blood glucose and oral candidal carriage in the saliva of type 2 diabetics: a case-control study," Contemporary clinical dentistry, vol. 5, no. 3, pp. 312-317, 2014.

[13] A. Man, C. N. Ciurea, D. Pasaroiu et al., "New perspectives on the nutritional factors influencing growth rate of Candida albicans in diabetics. An in vitro study," Memórias do Instituto Oswaldo Cruz, vol. 112, no. 9, pp. 587-592, 2017.

[14] C. Spampinato and D. Leonardi, "Candida infections, causes, targets, and resistance mechanisms: traditional and alternative antifungal agents," BioMed Research International, vol. 2013, 13 pages, 2013.

[15] T. Kadir, R. Pisiriciler, S. Akyuz, A. Yarat, N. Emekli, and A. Ipbuker, "Mycological and cytological examination of oral candidal carriage in diabetic patients and non-diabetic control subjects: thorough analysis of local aetiologic and systemic factors," Journal of Oral Rehabilitation, vol. 29, no. 5, pp. 452457, 2002.

[16] S. Al Mubarak et al., "The prevalence of oral_Candida_infections in periodontitis patients with type 2 diabetes mellitus," Journal of Infection and Public Health, vol. 6, no. 4, pp. 296301, 2013.

[17] C. M. P. D. C. Bianchi, H. A. Bianchi, T. Tadano et al., "Factors related to oral candidiasis in elderly users and non-users of removable dental prostheses," Revista do Instituto de Medicina Tropical de São Paulo, vol. 58, 2016.

[18] H. Nouraei, S. Sheykhi, Z. ZareShahrabadi, H. Khodadadi, K. Zomorodian, and K. Pakshir, "Comparative analysis of virulence factors of homozygous and heterozygous strains ofCandida albicansvaginal isolates," International Journal of Microbiology, vol. 2020, 5 pages, 2020.

[19] M. Manfredi, M. J. McCullough, Z. M. al-Karaawi, S. J. Hurel, and S. R. Porter, "The isolation, identification and molecular analysis of Candida spp. isolated from the oral cavities of patients with diabetes mellitus," Oral Microbiology and Immunology, vol. 17, no. 3, pp. 181-185, 2002.
[20] C. Tsang, F. C. S. Chu, W. K. Leung, L. J. Jin, L. P. Samaranayake, and S. C. Siu, "Phospholipase, proteinase and haemolytic activities of Candida albicans isolated from oral cavities of patients with type 2 diabetes mellitus," Journal of Medical Microbiology, vol. 56, no. 10, pp. 1393-1398, 2007.

[21] M. Fatahinia, F. Poormohamadi, and A. Z. Mahmoudabadi, "Comparative study of esterase and hemolytic activities in clinically important Candida species, isolated from oral cavity of diabetic and non-diabetic individuals," Jundishapur Journal of Microbiology, vol. 8, no. 3, p. e20893, 2015.

[22] M. Shimizu, N. Q. Almeida, V. Fantinato, and C. S. Unterkircher, "Studies on hyaluronidase, chondroitin sulphatase, proteinase and phospholipase secreted byCandidaspecies," Mycoses, vol. 39, no. 5-6, pp. 161-167, 1996.

[23] M. A. Ghannoum, "Potential role of phospholipases in virulence and fungal pathogenesis," Clinical Microbiology Reviews, vol. 13, no. 1, pp. 122-143, 2000.

[24] H. Nouraei, K. Pakshir, Z. ZareShahrabadi, and K. Zomorodian, "High detection of virulence factors by Candida species isolated from bloodstream of patients with candidemia," Microbial Pathogenesis, vol. 149, p. 104574, 2020.

[25] K. Zomorodian, F. Kavoosi, G. R. Pishdad et al., "Prevalence of oral Candida colonization in patients with diabetes mellitus," Journal de mycologie medicale, vol. 26, no. 2, pp. 103-110, 2016.

[26] M. F. Price, I. D. Wilkinson, and L. O. Gentry, "Plate method for detection of phospholipase activity inCandida albicans," Sabouraudia: Journal of Medical and Veterinary Mycology, vol. 20, no. 1, pp. 7-14, 1982.

[27] M. Slifkin, "Tween 80 opacity test responses of variousCandida species," Journal of Clinical Microbiology, vol. 38, no. 12, pp. 4626-4628, 2000.

[28] J. M. Manns, D. M. Mosser, and H. R. Buckley, "Production of a hemolytic factor by Candida albicans," Infection and Immunity, vol. 62, no. 11, pp. 5154-5156, 1994.

[29] L. M. Jaacks, K. R. Siegel, U. P. Gujral, and K. M. V. Narayan, "Type 2 diabetes: a 21st century epidemic," Best Practice \& Research Clinical Endocrinology \& Metabolism, vol. 30, no. 3, pp. 331-343, 2016.

[30] D. Mellitus, "Diagnosis and classification of diabetes mellitus," Diabetes Care, vol. 28, no. S37, pp. S5-S10, 2005.

[31] S. Shoham and S. Marwaha, "Invasive fungal infections in the ICU," Journal of Intensive Care Medicine, vol. 25, no. 2, pp. 7892, 2010.

[32] A. L. Lima, T. Illing, S. Schliemann, and P. Elsner, "Cutaneous manifestations of diabetes mellitus: a review," American Journal of Clinical Dermatology, vol. 18, no. 4, pp. 541-553, 2017.

[33] J. Premkumar, P. Ramani, T. Chandrasekar, A. Natesan, and P. Premkumar, "Detection of species diversity in oral candida colonization and anti-fungal susceptibility among non-oral habit adult diabetic patients," Journal of natural science, biology, and medicine, vol. 5, no. 1, pp. 148-154, 2014.

[34] C. Sriphannam, N. Nuanmuang, K. Saengsawang, D. Amornthipayawong, and A. Kummasook, "Anti-fungal susceptibility and virulence factors of Candida spp. isolated from blood cultures," Journal de Mycologie MÚdicale, vol. 29, no. 4, pp. 325-330, 2019.

[35] R. H. Bassyouni, A. A. Wegdan, A. Abdelmoneim, W. Said, and F. AboElnaga, "Phospholipase and aspartyl proteinase activities of Candida species causing vulvovaginal candidiasis in patients with type 2 diabetes mellitus," Journal of Microbiology and Biotechnology, vol. 25, no. 10, pp. 1734-1741, 2015. 
[36] K. Pakshir, K. Zomorodian, M. Karamitalab, M. Jafari, H. Taraz, and H. Ebrahimi, "Phospholipase, esterase and hemolytic activities of Candida spp. isolated from onychomycosis and oral lichen planus lesions," Journal de mycologie médicale, vol. 23, no. 2, pp. 113-118, 2013.

[37] G. Luo, L. P. Samaranayake, and J. Y. Yau, "Candida species exhibit differential in vitro hemolytic activities," Journal of Clinical Microbiology, vol. 39, no. 8, pp. 2971-2974, 2001. 